\title{
Jean Tirole, Poder de Mercado e a Nova Organização Industrial
}

\section{Jean Tirole, Market Power and the New Industrial Organization}

Carlos Alberto Cinquetti*

\begin{abstract}
Resumo: Conferido a Jean Tirole, um autor central da nova organização industrial (OI), o Nobel de Economia 2014 dá-nos oportunidade de uma ampla retrospectiva dessa nova área da microeconomia. Começamos com os estudos iniciais de oligopólio, avançando para estudos que levam a emergência da OI, e culminando com o novo arcabouço teórico de estratégias econômicas e incentivos sobre informações privadas. Nesta exposição que entrelaça o trabalho Tirole com a nova OI, damos uma atenção especial aos desenvolvimentos sobre contratos e incentivos. Ao considerar os impactos sobre o pensamento econômico, concentramo-nos em modelos não competitivos de comércio internacional, mais particularmente no largo uso de uma estrutura de mercado, concorrência monopolística, sem estratégias e sem diferenciação de qualidade dos bens.
\end{abstract}

Palavras-chave: Tirole. Poder de mercado. Regulação.

Abstract: The 2014's Nobel prize in Economics to Jean Tirole, an author so central for outlining the analytical frontier of modern industrial organization (IO), gives the opportunity for a comprehensive reassessment of this IO. This retrospective starts with the initial studies about oligopoly, moving to studies that lead to the establishment of the $\mathrm{IO}$, and culminates in the new theoretical framework for strategies and incentives under private information. The retrospective goes interwoven with Tirole's work and pays heed to the impact of these ideas upon recent economic thought. Upon considering applied fields, we focus on international trade, and discuss potentials and pitfalls of a deductive science.

Keywords: Tirole. Market power. Regulation.

JEL Classification: B3; D4; D8.

\section{1 lntrodução}

A nova organização industrial (OI) ganha corpo nos anos 1970 com a plena incorporação de decisões estratégicas (jogos) e abre várias novas fronteiras de pesquisa em teoria econômica, incluindo a nova teoria do comércio no início dos 1980. Com o posterior desenvolvimento da teoria dos jogos com informações privadas, fundando a teoria dos incentivos e contratos, abrem-se outras novas fronteiras na microeconomia. Nada mais expressivo, da dimensão desses avanços, que

Paulista Júlio de Mesquita (Unesp), campus de Araraquara. E-mail: cinquett@fclar.unesp.br 
metade dos prêmios Nobel em Economia de 1994 até o presente foi conferida às descobertas e formulações a partir de jogos e da nova OI.

O Nobel de 2014 dado a Jean Tirole pode ser visto como uma premiação para a nova OI. Tirole é francês, assim como os fundadores dos modelos de poder de mercado, Antonie Cournot e Josef Bertand, porém, diferentemente deles e de contemporâneos da OI agraciados com o Nobel - Schelling, Akerlof, Spence e Stiglitz e Maskin -, não se destaca por uma contribuição em particular, mas por um vasto conjunto de contribuições para a nova OI. ${ }^{1}$ Com uma carreira que lembra a do físico-matemático Poincaré, também formado na École Polytechnique, Tirole se notabilizou por contribuições originais e contemporâneas em vários temas, assim como por uma notável elegância expositiva. Os seus livros-texto intitulados The theory of industrial organization (1988), Game theory (1991) e A theory of incentives in procurement and regulation $(1993)^{2} \mathrm{o}$ tornaram o mais citado autor vivo da área e foram fundamentais para a demarcação analítica das novas fronteiras da OI. Esses livros foram, também, a porta de entrada principal para aqueles que aplicavam a OI em suas áreas de pesquisa. Seu prêmio Nobel é, dessa forma, uma excelente oportunidade para se fazer uma retrospectiva da nova OI, especialmente das análises sobre poder de mercado e regulação que lhe valeram o prêmio. O perfil de suas contribuições também nos permite discutir mais detidamente sobre certos avanços e tropeços da nova OI no comércio internacional.

\section{Primórdios, Ascensão e Morte da Ol}

Cournot (1838) e Bertrand (1883), com os modelos de oligopólio em quantidade e preços, respectivamente, estabelecem a análise de mercados não competitivos. Ao final do século XIX, Marshall (1890) integrou esses tópicos à análise positiva e normativa de mercados, definindo algumas condições e resultados gerais de ambos os mercados.

Sraffa (1926), expondo alguns dilemas das análises não competitivas, aponta uma das saídas: conceber outras dimensões além de preços e quantidades. Seguindo essa pista, Hotelling (1929) e Chamberlin (1933) avançam análises de concorrência por diferenciação de bens com modelos de concorrência oligopolista espacial e concorrência monopolística, respectivamente. ${ }^{3}$ Apenas após a Segunda

\footnotetext{
$1 \quad$ Ver Tirole (2014a).

2 Em outubro de 2014, o livro The theory of industrial organization (1988) figurava como o mais vendido manual avançado de OI no site da livraria norte-americana Amazon.

3 Solucionando o "paradoxo de Bertrand", assim como a hipótese de restrição da oferta (TIROLE, 1988, cap. 5).
} 
Guerra, com Mason (19394 apud PEPALL et al., 2008) e Bain (19565 apud PEPALL et al., 2008), estabelece-se um arcabouço analítico mais amplo e com forte apelo empírico, que definem o paradigma estrutura-conduta-performance (ECP). Funda-se, assim, a OI, voltada para firmas e mercados não competitivos.

O ECP integra as análises das seguintes questões: a) quanto uma estrutura de mercado se distancia de concorrência perfeita; b) qual a natureza da conduta dos agentes não tomadores de preços; e c) como a performance desses mercados se afasta da eficiência dos mercados competitivos.

O ECP teve imediata acolhida nos Estados Unidos, impulsionando o trabalho de agências reguladoras de competitividade e eficiência dos mercados. Entretanto, não resistiu a duas críticas associadas à escola de Chicago. A primeira foi em relação à noção de que, quanto mais concentrado um mercado, menos competitivo este será e pior também será sua performance. No entanto, evidências de integração vertical de firmas com redução no preço final dos produtos colocaram por terra o argumento. Como exposto por Tirole (1988), ainda que leve à monopolização na oferta de um insumo, a integração vertical evita o duplo mark-up sobre custos. Por vezes, o mercado contorna a monopolização dos insumos (TIROLE, 1988), segundo o espírito da escola de Chicago: pensar no ingresso e saída de firmas nos mercados, e não num mercado isoladamente.

O problema mais fundamental é que a formulação empírica de Mason e Bain partia de uma estrutura como um dado exógeno. Ainda que alguns trabalhos empíricos tenham tentado tratar a estrutura como endógena, ${ }^{6}$ a OI não estava teoricamente apoiada numa noção generalizável de comportamento dos agentes. A solução veio com o matemático John Nash, definindo uma formulação geral de equilíbrio em estratégicas (FUDENBERG; TIROLE, 1991), e essa aplicação de teoria dos jogos ao comportamento estratégico das firmas em mercados não competitivos conquista de vez a OI nos anos 1970 (TIROLE, 1988). É o marco zero da nova OI, com "conduta" definida a partir de decisões racionais dos agentes, condicionada às suas interações.

Ultrapassando uma teoria ad hoc para explicar fatos observados com uma nova formulação geral de decisões dos agentes, a OI ganha um guia mais seguro para intervenção governamental, ou regulamentação de mercados (TIROLE, 1988). Isto é, políticas fundadas em análises que consideram a reação dos agentes, o que implica uma perspectiva menos crédula a respeito dos cânones do mercado livre, como sugerido pela escola de Chicago. Análises que são, ao mesmo tempo, mais cautelosas, acerca da eficiência da intervenção estatal. ${ }^{7}$

4 MASON, E. S. Price and production policies of large scale enterprise. American Economic Review, v. 29, p. 61-74, 1939.

5 BAIN, J. Barriers to New Competition. Cambridge, MA: Harvard University Press, 1956.

6 Ver Tirole (1988).

7 A respeito, vale a leitura da entrevista de Tirole (ver TIROLE, 2014) logo após a premiação do Nobel. 
Pouco mais de 30 anos desde sua consolidação, pode-se dizer que a (nova) OI tornou-se a rainha da microeconomia aplicada, já que fez avançar modelos estruturais e de simulação que trazem as bases endógenas de estrutura e conduta (PERLOFF et al., 2007), sem desprezar explorações estatísticas mais simples - "deixar os dados falarem”, afinal métodos estatísticos raramente superam limitações das evidências disponíveis. Richard Schmalensee, colega de Tirole no MIT é, provavelmente, o mais destacado formulador da nova OI empírica. Pode-se dizer que a maioria dos especialistas em análise empírica tinha os livros-texto de Tirole como leitura de cabeceira acerca das hipóteses teóricas.

\section{Contratos e Incentivos}

Outra crítica da escola de Chicago ao ECP: se limitar a preços, quando muitas das relações das firmas vão além disso. A teoria dos incentivos e contratos, a partir de jogos sob informação privada, seria a resposta da nova OI a esse desafio. No entanto, o principal impulsionador dessa nova formulação foi, por um lado, o modelo de Akerlof $\left(1970^{8}\right.$ apud TIROLE, 1988) dos mercados de "micos" (ou "abacaxi”), que conduzirá aos modelos de seleção adversa, e, por outro, as análises de Willianson (1975) de ações oportunistas e procrastinadoras nas firmas, estendendo Coase (1937), que conduziram aos modelos de risco moral.

Quando qualidades e ações são observadas, modelos de preços trazem a solução contratual das transações, mas informações privadas (e.g., mercado de mico) sequer asseguram a existência de tais transações. Na relação de agência, esses jogos de informações incompletas são solucionáveis pela introdução de duas restrições: a participação e a compatibilidade nos incentivos. Até problemas tradicionais da OI, como descriminação de preços através de preços não lineares, encontram soluções mais satisfatórias quando redefinidos pelas relações de incertezas contidas, tomando consumidores como tipos escondidos (LAFFONT; TIROLE, 1993).

Incertezas e incentivos abrem grande espaço para se pensar em regulação e recontratação. O mais peculiar dessas formalizações é que a escolha de um arranjo institucional se torna a solução principal do problema, ao invés de preços e alocações para um dado arranjo (LAFFONT; TIROLE, 1993). Um exemplo é a elaboração de Weyl e Tirole (2012) sobre a remuneração socialmente ótima dos "direitos de propriedade" do inovador. Eles mostram que o mercado, ao revelar a disposição a pagar dos consumidores - privada ao inovador -, direciona para a eficiência econômica buscada pelo planejador, considerando a remuneração ótima do poder de mercado do inovador.

AKERLOF, G. The market for "lemmons": qualitative uncertainty and the market mechanism. Quarterly Journal of Economics, v. 89, p. 488-500, 1970. 
Na exposição sobre a teoria dos incentivos e contratos, Laffont e Tirole (1993) dão especial atenção aos monopólios naturais, base de atuação de órgãos governamentais. Esses estudos, a partir das teorias de contrato, são motivados pelas mudanças radicais no setor público nos anos 1980 e 1990, quer pelas privatizações de empresas estatais, quer pela reestruturação no modus operandi dos governos. São avançadas formulações normativas para formas de regulação, pela introdução ou modificação de incentivos que, atuando sobre as informações simétricas, estimulem a melhora de qualidade ou a queda nos preços dos serviços providos ou regulados pelo Estado. Infelizmente, no Sul do Equador, os insights da teoria dos incentivos para contratos e regulação governamental foram ignorados nos anos 1990, quando as discussões estavam polarizadas, em termos tradicionais, em torno do Consenso de Washington. No Brasil, pode-se dizer que os leilões de privatização, o estabelecimento das agências reguladoras, assim como o repensar sobre licitações públicas foram muito pouco orientados pelas proposições normativas a partir das teorias dos incentivos.

Certamente, uma das aplicações mais populares da teoria dos incentivos é a de Levitt e Dubner (2005), que mostra que a queda da criminalidade nas cidades americanas, a partir de meados dos anos 1990, não teria sido resultado da atuação mais dura da polícia - do prefeito Giuliani em Nova York - mas sim das leis de aborto, na virada dos anos 1970 para os 1980. Tais leis teriam acarretado a queda de nascimentos de crianças de mães (pobres) sem condições de cuidarem adequadamente de seus filhos. Levitt e Dubner (2005) abordam outras questões de economia do crime, cujos estudos estão muito enraizados nas noções de incentivos e informações assimétricas.

Incertezas e assimetria de informações são decisivas nos mercados financeiros, que, por isso mesmo, estão largamente amparados em relações contratuais explícitas. Tirole é um dos autores proeminentes na análise do setor financeiro a partir das teorias dos contratos, cujas falhas ou lacunas teriam precipitado a crise financeira de 2008 , os quais culminam no seu último livro-texto, The theory of corporate finance (2006). Numa resenha sobre o problema da iliquidez, Tirole (2011) dá um bom painel de como temas tradicionais de economia monetária e finanças são enriquecidos quando pensados a partir da teoria dos incentivos, isto é, quando pensando transações no mercado financeiro na perspectiva de escolha adversa, ou risco moral. ${ }^{9}$ Baseado na qualidade indefinida de ativos e passivos, são extraídas importantes proposições normativas, desde falhas nos índices de solvência em indicar risco de iliquidez, até regulamentações mais eficientes para se evitar crises de iliquidez.

$9 \quad$ Lembrando que a clássica análise de Stiglitz e Weiss (1981) sobre ajustes quantitativos no crédito por conta de tomadores com perfis incertos também deve ser pensada como análise em incentivos. 
Na teoria da firma é dominante a situação de risco moral, em que o esforço escondido do agente (gerente ou funcionário) impõe as restrições de participação e de compatibilidade dos incentivos. A economia dos direitos de propriedade suplantou a abordagem custo de transações, tratando-o como custo para definir, monitorar e impor direitos de propriedades. A percepção é a de que as fronteiras da firma não são definidas pelos custos de transação, mas pelos ativos específicos, o que garante direito de definir contratos e, assim, os modos de extração do excedente econômico entre principal e agente (HART; MOORE, 1990). Por exemplo, a internalização propiciada pela integração vertical não necessariamente reduz o custo de transação, mas a propriedade do capital físico confere ao proprietário o direito de definir quais partes da firma incorporada serão rearranjadas..$^{10}$

No caso das empresas multinacionais, ganha proeminência o capital conhecimento, cujos direitos de propriedade são difíceis de controlar e contratar. Mais precisamente, as frações de conhecimento explícito, $\sigma$, controlável, e de conhecimento tácito, 1 - $\sigma$, não controlável, são centrais para se definir um dos modos organizativos da firma do país $N$ que decida produzir no país $S$ : em transações internas à firma (empresas multinacionais), ou via licenciamento (terceirização). Nesse último caso, o agente externo controla o capital físico, o que lhe garante uma barganha superior na extração do excedente da empresa. Assumindo uma proporcionalidade da razão capital conhecimento/capital físico com $\sigma$, Chen et al. (2012) deduzem que a empresa multinacional será o arranjo mais característico em setores intensivos em capital conhecimento e baixa escala. Em setores de alta escala - indicadora da intensidade de capital físico - e baixo $\sigma$, cai o risco e o valor de protelações (hold up) por parte do agente, por conta de seu controle de propriedade, sob terceirização, que passa a ser mais rentável.

\section{Organização Industrial e Comércio Internacional}

Como indicado por Tirole (1988), a nova teoria do comércio (NTC) foi uma espécie de filha predileta da nova OI. Evidentemente, os estudos iniciais sobre poder de mercado e políticas (regulamentações) comerciais envelheceram, e os novos, que vão além da NTC, e propõem pensar novos temas sob contratos, seguindo a própria trajetória da OI, que envelhecem. Antes de oferecer um painel geral desses estudos, focaremos num extremo apego à concorrência monopolística, por parte dos economistas de comércio internacional, em formulações incongruentes e contraditórias a nova OI.

O equilíbrio de Nash dá maior generalidade aos equilíbrios de Cournot e de Bertrand. Da conjectura Cournot-Nash, por exemplo, se deduz que um mercado internacional integrado traria um efeito pró-competitivo mesmo que, ausentes

10 Pensa-se em integração vertical numa perspectiva diferente da discutida na seção anterior. 
vantagens comparativas, não haja comércio internacional (MARKUSEN, 1981). Porém, as mais impactantes extensões da OI para a nova teoria do comércio vieram de modelos de concorrência monopolística. A partir de Spence (1976), Krugman $(1979,1980)$ deduz o efeito variedades, de modo que a ineficiência do poder de mercado é contrabalanceada por ganhos aos consumidores. De Dixit e Stiglitz (1977), Krugman deduz os padrões de comércio inter e intraindústria - o paradigma Krugman-Dixit-Stiglitz (KDS) - ampliando a concorrência monopolística para uma análise de equilíbrio geral em quantidades e variedades.

Essa transposição da OI para análises de equilíbrio geral explica a larga acolhida do modelo KDS em comércio internacional. Trata-se de um modelo no qual, além de um nível único de qualidade, a ausência de inteirações estratégicas - preços e custo marginal têm uma relação constante - facilita análises de um equilíbrio geral internacional. Porém, o que se teve foi uma meia nova teoria de comércio, ${ }^{11}$ ou duas e meia, se considerarmos desenvolvimentos baseados em oligopólio, ou nas novas formulações de incentivos.

Os excessos com concorrência monopolística estão patentes tanto na hegemonia de mais de um quarto de século do KDS, quanto em sua superação por um modelo (MELITZ, 2003) que apenas introduz firmas heterogêneas e custo fixo de comércio, sem alterar os elementos microeconômicos de um markup exógeno. Outros sintomas incluem extrapolações empíricas, como atribuir à concorrência monopolística padrões de comércio bilateral (HELPMAN, 1987) que remetiam ao comércio gravitacional num modelo competitivo. Por outro lado, houve explorações geniais dessas características comuns ao modelo competitivo, como o quase-Heckscher-Ohlin de Romalis (2004), deduzindo comércio intra e interindústrias a partir de diferenças tecnológicas e dotações de fatores num mundo com vários países.

Melitz e Ottaviano (2008) reintroduzem inteirações estratégicas, mas o suposto de um contínuo de firmas no setor impossibilita discriminar domínio de mercados por poucas empresas. A ignorada concorrência monopolística espacial de Lancaster (1979, 1984), inspirada em Hotelling, resolve, mas não gera soluções analíticas com firmas heterogêneas. Neary (2010), sob certas condições de ingresso das firmas, supera essas dificuldades com num oligopólio Cournot para análise de equilíbrio geral.

A concorrência monopolística foi também aplicada, em comércio internacional, para pensar concorrência em qualidades de bens, quando Tirole (1988) já havia enfatizado que o modelo trata apenas do grau de diversificação de bens, e não de qualidades. Schott (2004) também observa, sem remeter à literatura de competição em qualidade da OI, que as firmas líderes vendem bens tão ou mais caros que seus concorrentes, enquanto Melitz (2003) prevê que as firmas mais competitivas seriam maiores porque venderiam a preços mais baixos.

11 Alcunha do próprio Paul Krugman, em manifestação informal a Peter Neary (2010). 
O suposto de concorrência monopolística traz dificuldades inexoráveis em estudos empíricos posteriores que avançaram sobre comércio em qualidade de bens. Hallak e Schott (2011), por exemplo, trazem uma demanda por qualidade que não é derivada de otimização sob restrição de preferências em qualidade, enquanto a forma CES de preferências compostas por qualidade e quantidades de Kluger e Verhoogen (2011) conduz a uma demanda homotética por qualidade, suprimindo as ligações de demanda.

O rumo desses desenvolvimentos surpreende quando lembramos que a fronteira de comércio internacional na qualidade de bens abre-se com Rauch (1999), cuja análise empírica parte de três grupos de bens: diferenciados, referenciais e não diferenciados. A variação de preços dos diferenciados à distância (ou ligações culturais) indica uma estrutura de mercado não associável à diferenciação horizontal. Numa estrutura oligopolista, Eckel et al. (2013) conseguem articular oferta de qualidade a marketing, enquanto o modelo ricardiano, com preferências não homotéticas de Fieler (2011a, 2011b), consegue derivar as direções internacionais da demanda por qualidade conforme renda dos consumidores. Como indicado em Hummels e Klenow (2005), o recurso à concorrência monopolística ${ }^{12}$ era como que um expediente facilitador, de usar o cavalo de batalha. Mas isso significa "modelar por força do hábito" que, como se viu, resultou numa incongruência entre modelo (de diferenciação horizontal) e objeto (de diferenciação vertical). Ademais, trata-se de formulações ad hoc para qualidade, como se os modelos de comércio internacional apoiados em concorrência imperfeita sem inteiração estratégica fossem culminar no que foi o ponto de partida, ou de ruptura da nova OI: as hipóteses ad hoc.

Podemos falar em limites da ciência não pelo estágio do conhecimento científico, mas sim pelos limites do trabalho científico em lidar com o conhecimento disponível, em parte, por uma má especialização do trabalho nesses estudos em comércio internacional. Primeiro por não remeter temas da OI a especialistas de OI, ${ }^{13}$ contradizendo tendências de ampliação do leque de especialistas envolvidos nas pesquisas. Segundo, pela ampliação do escopo dos trabalhos, que passaram a combinar modelagem matemática e prova empírica. Ainda que inspirada ou testada em pesquisas empíricas, a modelagem teórico-dedutiva nas ciências se sustenta por si própria. ${ }^{14}$

12 Como ilustrado no trabalho de Hallack (COIBON et al., 2007) - um autor com raro e avançado domínio de OI -, que formula, num modelo puro de OI, diferenciação horizontal e vertical de bens.

13 Sobre a colaboração com especialistas da OI cada vez menos frequente, ver o perfil dos autores na coletânea de Grossman (1992).

14 Vale lembrar a física nos seus primórdios, quando as colaborações no trabalho científico eram menores: o modelo teórico para a órbita elíptica dos planetas de Kepler se apoiou nas observações de Tycho, enquanto o modelo de ondas eletromagnéticas de Maxwell se apoiou nas observações de Faraday. 
A julgar por Acemoglu et al. (2014), além do próprio Nobel 2014 de economia, pode-se prever que os estudos de concorrência por qualidade aumentará o espaço de modelos com poder de mercado endógeno e com um número discreto de firmas. Por outro lado, esse modelador teórico da OI com enorme senso aplicado, Tirole, pode estimular os economistas de comércio a repensarem o escopo de seus artigos, separando modelagem teórica e empírica. Isso facilitaria a colaboração com autores de áreas diversas, como os da OI. É um desafio para as pesquisas em comércio internacional não baseado em vantagens comparativas: revalorizar as vantagens comparativas dos especialistas envolvidos.

\section{Considerações Finais}

Aproveitando os avanços da matemática, a OI conseguiu responder a desafios surgidos desde seu início: superou a modelagem ad hoc, formulando a decisão das firmas a partir de uma teoria geral de decisões num ambiente de inteirações; superou, uma proporcionalidade definida e geral entre grau de concentração (estrutura) e competitividade (conduta); e, apoiada em jogos no paradigma de agências, formulou inteirações de uma forma institucional mais rica, indo além de preços.

Esses avanços na modelagem teórica foram acompanhados por novas modelagens empíricas. Não obstante, em modelos de comércio internacional não competitivo, o desafio de combinar a OI e o equilíbrio geral envolveu desafios próprios e ingentes. Em alguns casos, a pressão por respostas rápidas foi resolvida recorrendo-se a adaptações de "sagrados cavalos de batalha", que eram incoerentes com o tema posto.

\section{Referências}

ACEMOGLU, D.; LAIBSON, D.; LIST, J. Microeconomics. The USA: Pearson. 2014.

BERTRAND, J. Théorie Mathématique de la Richese Sociale. Journal des Saunts, p. 499-508, 1883.

BOCCARD, N. Industrial organization: a contract-based approach. Spain: University of Girona, 2010. Disponível em: <http://nboccard.wordpress.com/> Acesso em: 10 jul. 2011.

CHAMBERLIN, E. The Theory of Monopolistic Competition. Cambridge, MA: Harvard University Press. 1933

CHEN, Y.; HORSTMANN, I. J.; MARKUSEN, J. R. Physical capital, knowledge capital, and the choice between FDI and outsourcing. Canadian Journal of Economics, v. 45, n. 1, p. 1-15, 2012.

COASE, R. H. The nature of the firm. Economica, v. 4, p. 386-405, 1937.

COIBION, O.; EINVAV, L.; HALLAK. J. C. Equilibrium demand elasticities across quality segments. International Journal of Industrial Organization, v. 25, p. 13-30, 2007. 
COURNOT, A. Reserches sur les Principes Mathématiques de Théorie des Richeses. 1838. English edition (ed. N. Bacon), Researches into the Mathematical Principles of the Theory of Wealth. New York: Macmillan, 1897.

DIXIT, A.; STIGLITZ, J. Monopolistic competition and optimum product diversity. American Economic Review, v. 67, p. 297-308, 1977.

EATON, J.; KORTUM, S. Technology, geography, and trade. Econometrica, v. 70, n. 5, p. 1741-1749, 2002.

ECKEL, C. et al. Multi-product firms at home and away: cost- versus quality-based competence. University of Oxford and CEPR, 2013. Acesso em: <http://users.ox.ac.uk/ econ0211/papers/ pdf/multipro2.pdf > . Acesso em: 07 dez. 2014.

FIELER, A. C. Nonhomotheticity and bilateral trade: evidence and a quantitative explanation. Econometrica, v. 79, n. 4, p. 1069-1101, 2011 a.

. Quality differentiation in trade: theory and evidence. Philadelphia, PA: Department of Economics, University of Pennsylvania, 2011b. (Working paper).

FUNDENBERG, D.; TIROLE, J. Game theory. Cambridge: The MIT Press, 1991.

GROSSMAN, G. (Ed.). Imperfect competition and international trade. Cambridge: The MIT Press, 1992.

HALLAK, J. C.; SCHOTT, P. Estimating cross-country differences in product quality. The Quarterly Journal of Economics, v. 126, n. 1, p. 417-474, 2011.

HART, O.; MOORE, J. Property rights and the nature of the firms. Journal of Political Economy, V. 98, n. 6, p. 1119-1158, 1990.

HELPMAN, E. Imperfect competition and international trade: evidence from fourteen industrial countries. Journal of the Japanese and International Economics, n. 1, p. 62-81, 1987.

HELPMAN, E.; KRUGMAN, P. R. Market structure and foreign trade. Cambridge: The MIT Press, 1985.

HOTELLING, H. Stability in Competition. Economic Journal, 39, 41-57.

HUMMELS, D.; KLENOW, P. J; The variety and quality of a nation's exports. The American Economic Journal, v. 95, n. 3, p. 704-723, 2005.

KLUGER, J.; VERHOOGEN, E. Prices, plant size, and product quality. The Review of Economic Studies, v. 79, p. 307-339, 2012.

KRUGMAN, P. R. Increasing returns, monopolistic competition, and international trade. The Journal of International Economics, v. 9, n. 4, p. 469-479, 1979.

Scale economies, product differentiation, and the pattern of trade. American Economic Journal, v. 70, n. 5, p. 950-59, 1980. 
LAFFONT, J. J.; TIROLE, J. A theory of incentives in procurement and regulation. Cambridge: The MIT Press, 1993.

LANCASTER, K. Protection and product differentiation. In: KIERZKOWSKI, H. (Ed.). Monopolistic competition and international trade. Oxford: Claredon Press, 1984, p. 137-156.

. Variety, equity, and efficiency. New York: Columbia University Press, 1979.

LEVITT, S. D.; DUBNER, S. P. Freakonomics. Rio de Janeiro: Ed. Campus, 2005.

MARKUSEN, J. R. Trade and the gains from trade with imperfect competition. Journal of International Economics, v. 11, n. 4, p. 531-551, 1981.

MARSHALL, A. Principles of Economics. London: Macmillan and Co., 1890. Edição português: Princípios de Economia. São Paulo: Abril Cultural. 1982.

MELITZ, M. J. The impact of trade on intra-industry reallocations and aggregate industry productivity. Econometrica, v. 71, n. 6, p. 1695-1725, 2003.

MELITZ, M.; OTTAVIANO, G. Market size, trade, and productivity. Review of Economic Studies, v. 75, n. 1, p. 295-316, 2008.

NEARY, J. P. Two and a half theories of trade. 2010. (CEPR discussion papers, n. 7600)

PEPALL, L.; RICHARDS, D.; NORMAN, G. Industrial organization. U.K.: Blackwell, 2008.

PERLOFF, J. M.; KARP, L. S.; GOLAN, A. Estimating market power and strategies. New York: Cambridge University Press, 2007.

RAUCH, J. E. Networks versus market in international trade. Journal of International Economics, v. 48, n. 1, p. 7-35, 1999.

ROMALIS, J. Factor proportions and the structure of commodity trade. The American Economic Review, v. 94, n. 1, p. 67-97, 2004.

SCHOTT, P. K. Across-product versus within-product specialization in international trade. The Quarterly Journal of Economics, v. 119, n. 2, p. 647-678, 2004.

SPENCE, M. Product selection, fixed costs, and monopolistic competition. The Review of Economic Studies, v. 43, n. 2, p. 217-235, 1976.

SRAFFA, P. The laws of return under competitive conditions. Economic Journal, 36: 535-550, 1926.

STIGLITZ, J. S.; WEISS, A. Credit rationing in markets with imperfect information. American Economic Review, v. 71, p. 393-410, 1981.

TIROLE, J. Liquidity and all its friends. Journal of Economic Literature, v. 49, n. 2, p. 287-325, 2011.

. Long CV. Disponível em: <https:/www.tse-fr.eu/sites/default/files/TSE/documents/

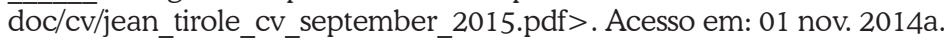


Reguladores precisam estar prontos para intervir. In: Estadão, São Paulo, 21 out. 2014. Disponível em: <http://economia.estadao.com.br/noticias/geral,reguladoresprecisam-estar-prontos-para-intervir-imp-,1580039>. Acesso em: 21 out. 2014b.

. The theory of corporate finance. New Jersey: Princeton University Press, 2006.

. The theory of industrial organization. The MIT Press: Cambridge, 1988.

WEYL, E. G.; TIROLE, J. Market power screens willingness-to pay. Quarterly Journal of Economics, v. 4, p. 1971-2003, 2012.

WILLIAMSON, O. E. Markets and hierarchies: analysis and antitrust implications. New York: The Free Press, 1975.

Recebido em: 11/11/2014. Aceito em: 13/03/2015. 